\title{
Desórdenes orales potencialmente malignos. Lo que el odontólogo debe conocer
}

\author{
Oral potentially malignant disorders-What the dentist should know
}

Marcos Felipe Iparraguirre Nuñovero ${ }^{1, a}$, Ximena Fajardo ${ }^{2, b}$, Everdan Carneiro ${ }^{1, c}$, Paulo Henrique Couto-Souza ${ }^{1, d}$

\section{RESUMEN}

Objetivo: El objetivo de este estudio es actualizar los conocimientos sobre Desordenes Orales Potencialmente Malignos, dando énfasis a datos epidemiológicos, apariencia clínica, sintomatología, diagnóstico diferencial y manejo terapéutico, así como también resaltar el rol del odontólogo general en el examen clínico. Material y métodos: Fue realizada una búsqueda de la literatura en las bases de datos científicas "Medline", "Pubmed" y "SciELO", utilizando las palabras clave "Leukoplakia", "Erythroplasia", "Cheilitis", "Nevus, Pigmented", "Oral Submucous Fibrosis", "Lichen Planus". La búsqueda fue orientada hacia artículos de revisión, estudios clínicos y reportes de caso, priorizando los artículos publicados en los últimos 5 años y sin hacer distinciones por el idioma. Resultados: Como resultado de la búsqueda se obtuvieron 165 artículos, los cuales fueron revisados cuidadosamente para conservar solo aquellos que se dedicaran a describir, comparar o tratar desordenes con potencial de malignidad, incluyendo datos de relevancia como epidemiología, apariencia clínica, sintomatología, diagnóstico diferencial y manejo terapéutico. De esta manera, el estudio se limitó a 41 artículos. Conclusiones: Al adoptar medidas preventivas, así como un meticuloso examen clínico sobre tejidos blandos, se puede facilitar la detección temprana de Desordenes Orales Potencialmente Malignos. La eliminación de lesiones sospechosas o un control de las condiciones cancerígenas puede evitar en gran mayoría el desarrollo de tumores malignos.

Palabras clave: Enfermedades de la boca, patología bucal, leucoplasia bucal, neoplasias de la boca, lesiones precancerosas.

\section{SUMMARY}

Objective: The objective of this study is to update scientific knowledge about OPMDs, emphasizing epidemiological data, clinical appearance, symptomatology, differential diagnosis and therapeutic management, as well as highlighting the role of the general dentist in the clinical examination. Material and methods: A literature search was carried out, making use of the keywords "Leukoplakia", "Erythroplasia", "Cheilitis", "Nevus, Pigmented", "Oral Submucous Fibrosis" and "Lichen Planus". Prioritizing articles published in the last 5 years on the scientific databases "Medline", "Pubmed" and "SciELO". Results: As a result of the search, 165 articles were obtained, which were carefully reviewed to preserve only those that were dedicated to describe, compare or treat lesions with malignant potential, including relevant data such as epidemiology, clinical appearance, symptomatology, differential diagnosis and management. therapeutic. In this way, the study was limited to 41 articles. Conclusions: By taking preventive measures, as well as through a clinical examination of soft tissues, early detection of potentially malignant disorders can be facilitated. The removal of suspicious lesions or a control of their carcinogenic conditions can largely prevent the development of malignant tumors.

Key words: Pathology, oral, leukoplakia, mouth neoplasm, precancerous conditions.

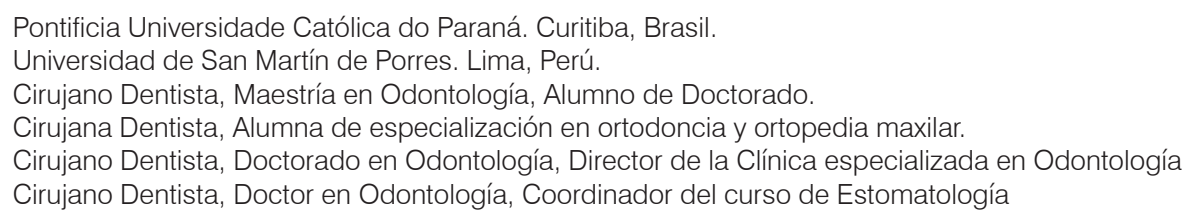




\section{INTRODUCCIÓN}

La Odontología ha pasado por muchas etapas al correr de los años para poder llegar a lo que es hoy. El concepto de solo tratar lo que un paciente aqueja quedó en el pasado ante la aparición de diversos desórdenes orales los cuales pueden desarrollarse bajo silencio clínico, es decir, sin presentar ningún tipo de sintomatología para el paciente. La Odontología moderna debe basarse en un principio de prevención constante, donde el clínico debe cumplir protocolos estrictos dentro del examen intraoral, evaluando minuciosamente tanto tejidos blandos como tejidos duros y estar preparado ante la presencia de algún tipo de lesión sospechosa, ya sea para tratarla o derivarla si fuera el caso correspondiente.

La cavidad oral puede verse afectada como cualquier otra región de nuestro organismo por Neoplasias Malignas (NM), las cuales en algunos casos son precedidas por Desórdenes Orales Potencialmente Malignos (DOPMs) $(1,2)$.

Los DOPMs son alteraciones sobre el tejido oral, las cuales pueden asumir características de tumor maligno y posteriormente transformarse en cualquier momento. Existe un incremento considerable de la incidencia de DOPMs en el último siglo, esto se debe a la influencia de factores externos perjudiciales para la salud oral como el consumo de tabaco, alcohol, betel quid y la exposición a la radiación solar $(3,4)$.

Una detección temprana de estas lesiones es de suma importancia para prevenir el desarrollo de entidades malignas como lo es el Carcinoma Oral de Células Escamosas (COCE). El cual, en la mayoría de los casos, es detectado de manera tardía, etapa asociada a un peor pronóstico y calidad de vida. Un diagnóstico y tratamiento de una lesión en etapa precancerosa, sin duda mejoraría las tasas de supervivencia $(5,6)$.

La presente revisión de la literatura tiene como objetivo actualizar los conocimientos sobre DOPMs del odontólogo general, dando énfasis a datos epidemiológicos, apariencia clínica, sintomatología, diagnóstico diferencial y manejo terapéutico de cada lesión. Si bien esta revisión brinda una noción sobre el tratamiento de las lesiones, debemos aclarar que el manejo de estas lesiones se encuentra dentro de las competencias de especialistas del área de Patología Oral y de Cirugía Buco Maxilofacial. El odontólogo general cumple un rol importante en la detección temprana de la lesión, así como también en informar y direccionar al paciente.

\section{MATERIAL Y MÉTODOS}

Para esta revisión de la literatura fueron utilizadas las palabras clave "Leukoplakia", "Erythroplasia", "Cheilitis", "Nevus, Pigmented", "Oral Submucous Fibrosis", "Lichen Planus", previamente consultadas en página "DeCS -Descriptores en Ciencias de la Salud": http://www.decs.bvs.br/. Para la búsqueda también fueron empleados operadores booleanos con la finalidad de crear una llave de búsqueda más específica para cada una de las lesiones revisadas, y así limitar los resultados obtenidos. La búsqueda de literatura fue realizada en las bases de datos "Medline", "Pubmed" y "Scielo", priorizando los artículos publicados en los últimos 5 años y sin hacer distinciones por el idioma. Se revisaron también 4 libros actualizados en estomatología, patología y medicina oral.

\section{RESULTADOS}

Como resultado de la búsqueda se obtuvieron 165 artículos, los cuales fueron revisados cuidadosamente para conservar solo aquellos que se dedicaran a describir, comparar o tratar lesiones con potencial de malignidad, incluyendo datos de relevancia como epidemiología, apariencia clínica, sintomatología, diagnóstico diferencial y manejo terapéutico. De esta manera, el estudio se limitó a 41 artículos científicos. Se presenta la información recopilada tratando a mayor detalle cada desorden o lesión oral con un considerable potencial de malignidad, así como también las opciones de manejo terapéutico.

\section{Análisis e integración de la información Leucoplasia}

La OMS define a la Leucoplasia como una placa blanca de la mucosa oral que no puede ser removida por raspaje y que no puede ser caracterizada clínica o patológicamente como cualquier otra enfermedad. Es un término estrictamente clínico y no implica una alteración histopatológica específica $(4,7,8,9)$.

Es el DOPM más común de la mucosa oral representando el 85\% entre estas. (8) Antiguamente existía un gran predominio sobre pacientes varones en compa- 
ración a mujeres. Sin embargo, según Carrard, debido al incremento en el último siglo de hábitos como fumar y el consumo de alcohol en pacientes femeninas, este predominio se ha equilibrado siendo actualmente tanto hombres como mujeres casi igualmente afectados (7). Se calcula una prevalencia entre 1,72 $-2,74 \%$ y un potencial maligno del $2-4 \%$ de los casos. Existen algunos factores para medir la gravedad y el riesgo de transformación como lo es el tamaño de la lesión, la localización y la presencia o no de displasia epitelial $(7,10)$.

Según Neville, más el 70\% de los casos de Leucoplasia aparecen a nivel de la lengua y bermellón del labio. Sin embargo, Regezi señala que actualmente casi la mitad de los casos reportados de Leucoplasia involucran también áreas como la mucosa alveolar mandibular y a la mucosa yugal $(8,9)$.

Clínicamente se divide en dos subtipos, lesiones homogéneas y no homogéneas:

Homogéneas: se caracterizan por tener una apariencia de placa blanca uniforme y bien delimitada en al menos una porción de esta, además de verse delgada y sin una elevación diferenciada pudiendo o no tener fisuras a lo largo de la lesión.

No homogéneas: Consideradas las más peligrosas, se caracterizan por tener presencia de un infiltrado rojo que se observa en forma de moteado dentro de la lesión blanca. Momento en el cual se utiliza el término de "Leucoplasia Mosqueada" o "Eritroleucoplasia". La Leucoplasia Verrugosa Proliferativa es otro tipo de leucoplasia no homogénea. Suele afectar más a mujeres y se encuentra íntimamente asociada al consumo de tabaco. Se caracteriza por presencia de nódulos/verrugas a lo largo de la lesión las cuales progresan y se diseminan a las áreas continuas de su aparición, siendo la encía la zona afectada con mayor frecuencia. Posee una tasa de potencial de transformación maligna del $74 \%$ junto a un porcentaje de recurrencia entre $87-100 \%$ de los casos $(1,11,12)$.

Un trabajo de revisión sistemática realizado por Warnakulasuriya en el año 2016 concluyó que, a pesar de ser una lesión relativamente más frecuente en pacientes masculinos, son las pacientes femeninas quienes corren un significante mayor riesgo de transformación maligna de la lesión. También fue concluido que la leucoplasia de tipo no homogénea tiene un mayor riesgo de transformación(10).
Se debe tomar mucho cuidado y atención al momento de examinar una lesión sospechosa de leucoplasia debido al gran parecido clínico que guarda con otros diagnósticos. Otros desórdenes como liquen plano, queratosis friccional, queratosis del fumador, estomatitis nicotínica, leucoedema y nevus blanco esponjoso deben ser excluidos clínicamente antes de dar un diagnóstico de leucoplasia (8). Incluso, diferentes tipos de leucoplasias pueden exhibir características clínicas similares entre sí, pero presentan un considerable grado de heterogeneidad microscópica que las diferencia entre sí. Su aspecto microscópico puede variar entre una hiperqueratosis a un COCE. Por esta razón, la realización de una biopsia es mandataria para el establecimiento del diagnóstico definitivo $(4,9)$.

El tratamiento es guiado según el tamaño de la lesión. En caso la lesión no presente displasia epitelial al examen histopatológico, se puede aplicar controles sobre el paciente intentando cesar el hábito de fumar y retirando cualquier otro factor irritante sobre la lesión. Caso exista una displasia epitelial, principalmente severa, el tratamiento indicado es la remoción quirúrgica de la lesión y de igual manera corregir hábitos o factores irritantes. Lo esencial es que la lesión sea removida en su totalidad junto con un pequeño margen de seguridad tanto en extensión y profundidad $(8,9)$. Los estudios revisados para este trabajo revelan que tanto el tratamiento quirúrgico como el no quirúrgico, no siempre son del todo efectivos para prevenir una posible transformación maligna o una reaparición de la lesión. Por este motivo, se le recomienda al odontólogo mantenerse alerta después del tratamiento, es importante realizar controles periódicos semanales o mensuales, dependiendo de la gravedad de la lesión $(1,8,9,10,12)$.

\section{Eritroplasia}

La eritroplasia oral es un DOPM poco común considerado como una de las lesiones con mayor riesgo a malignizarse. Fue descrita por primera vez por Queyrat en 1911, según estudios, tiene una prevalencia del $0.02 \%$ al $0.83 \%$ (13). Entre las áreas más afectadas encontramos a la mucosa bucal, la mucosa palatina y la mucosa yugal $(14,15,16)$. Según Kalavrezos, esta lesión puede afectar a ambos sexos por igual, teniendo un mayor porcentaje de aparición a partir de la sexta y séptima década de vida(1).

Entre los factores principales que propician el origen de esta lesión, se encuentran hábitos como fumar, masticar tabaco, masticar betel quid y la ingesta de 
alcohol $(1,16)$.

Un estudio realizado por Nielsen et al., en 1996, mostró resultados donde 5 de cada 10 casos de pacientes con eritroplasia presentaron un resultado positivo al virus del papiloma humano (VPH) (17). Además, aseguró que todos los casos de eritroplasia que posteriormente alcanzaron a desarrollar una neoplasia maligna fueron positivos a este virus, formando así la hipótesis que relaciona al VPH con las lesiones precancerosas.

Clínicamente, la eritroplasia es una lesión de tipo mancha bien definida, de color rojo brillante con una superficie que puede ser lisa, aterciopelada o granular $(13,14,18)$. Histológicamente, el epitelio es atrófico y delgado, y está caracterizado por su falta de queratina (16). Es muy frecuente la presencia de cambios epiteliales que van desde una displasia epitelial hasta un Carcinoma in situ (13).

Las lesiones con infiltrado rojo tienen una mayor tendencia de malignizarse en comparación a las lesiones blancas, en vista de esto se debe llevar un control riguroso sobre los pacientes que presenten estas características $(14,19,20)$. El clínico general suele confundir la eritroplasia con candidiasis eritematosa o con eritema migratorio, dentro del diagnóstico diferencial deben descartarse lesiones como liquen plano, lupus eritematoso y otros desórdenes erosivos (18).

El diagnóstico definitivo se debe confirmar mediante la biopsia de la lesión. De confirmarse el diagnóstico, la resección quirúrgica completa será el tratamiento de elección. Esta resección incluye el extirpar la lesión conteniendo displasia epitelial grave o carcinoma in situ $(16,18)$.

\section{Queilitis actínica}

La queilitis actínica es una lesión crónica potencialmente maligna de los labios, considerada como una manifestación temprana del carcinoma de células escamosas $(21,22,23)$.

Afecta principalmente al sexo masculino de raza blanca a partir de la cuarta década de vida y tiene un potencial maligno que oscila entre $11-36 \%(24,25)$. Según Ito, el $95 \%$ de los casos se localizan a nivel del labio inferior, esto se debe a la delgadez de su epitelio y a la falta de melanina y queratina que lo hacen más susceptible ante la exposición de rayos ultravioleta (23).

Todos los estudios coinciden en que la principal causa que favorece la aparición de esta lesión es la radiación ultravioleta originada por la exposición crónica al sol (21, 23, 24, 25), sin embargo, Hernández Osorio describe otros factores de riesgo que también deben ser tomados en cuenta dentro de la etiopatogenia de esta lesión. Entre los factores que la autora menciona, encontramos el fototipo (Capacidad para asimilar la radiación), el consumo de tabaco, la edad, la ocupación, el sexo y el consumo de alcohol (25).

Los pacientes con queilitis actínica presentan clínicamente pérdida de los bordes del labio inferior, acompañado de un oscurecimiento de la zona que limita el labio con la piel. Estos signos suelen ser acompañados de ulceraciones y descamaciones. Dentro de los síntomas se incluyen el dolor, picazón y sequedad de boca $(23,25,26)$. Histológicamente, hay presencia de factores como hiperqueratosis, hipercromatismo nuclear, atrofias, cambios citológicos, mitosis aumentadas, displasia epitelial, entre otros $(23,24,25)$.

Como ya fue mencionado anteriormente, no siempre existe relación exacta entre la parte clínica y la histológica. Por este motivo el diagnóstico definitivo debe darse a través de una biopsia incisional (25). El tratamiento para la queilitis actínica comienza por instruir al paciente a tomar medidas de protección contra los rayos UV, ya sea mediante el correcto uso de protectores solares o utilizando vestimenta adecuada (22, 25). Choi recomienda un manejo farmacológico tópico mediante el uso de 5-fluorouracilo o diclofenaco (21). Finalmente se puede optar por una intervención quirúrgica incluyendo procedimientos como la crioterapia, ablación con láser, vermilionectomía o terapia fotodinámica $(21,23,24)$.

\section{Nevus pigmentar}

El Nevus es considerado una anomalía del desarrollo en la piel presente en la gran mayoría de las personas. Se clasifica según su variedad histológica pudiendo ser de tipo "unión", "compuesto" o "intradérmico" $(4,9,27)$.

A pesar de ser la lesión de origen melanocítico más común, específicamente las de tipo unión son muy infrecuentes en mucosa oral, llegando a estar presente en el $0,9 \%$ de los casos, siendo las personas en un rango de edad entre 30 a 40 años las más afectadas (28). Clínicamente se observan pápulas elevadas $(<0,5 \mathrm{~cm})$ que en el $20 \%$ de los casos no se ven pigmentadas. Cualquier parte de la mucosa puede verse afectada siendo las zonas más comunes el paladar, encías y labio inferior. Una variación repentina de la intensidad de la pigmentación o presencia de sangrado debe ser considerado como una señal de riesgo de transformación maligna $(9,29)$. 
Según Regezi, la transformación maligna de un nevus oral es altamente improbable, el autor menciona que no hay suficientes casos o evidencia que pueda comprobar una transformación maligna, y sugiere que el cuidado que se tiene con esta lesión se debe al parecido que guarda con otras lesiones como lo puede ser un melanoma en etapa temprana (9). Sin embargo, en el 2015 Liang reportó un caso de un hombre de 48 años, el cual presentó un nevus, de tipo de unión, el cual atravesó una transformación para finalmente convertirse en un melanoma maligno, Liang concluye que cuando el nevus se ve frente a una infección crónica, agente irritante o algún otro estímulo agresivo constante se origina una hiperactividad melanocítica la cual podría llevar al nevus a transformarse (29).

Se sugiere sospechar de todos los nevus orales con un tamaño mayor a $1 \mathrm{~cm}$. De confirmarse el diagnóstico mediante biopsia, se recomienda remover quirúrgicamente el nevus, debido a que es difícil evitar irritación crónica sobre la lesión $(9,29)$.

\section{Fibrosis submucosa oral}

La Fibrosis Submucosa Oral (FSO) es un trastorno potencialmente maligno de progresión lenta y alto riesgo, la cual se caracteriza por la formación de bandas fibrosas a lo largo de la mucosa oral y que ocasionalmente pueden extenderse hasta la faringe y primer tercio del esófago. Clínicamente se observan zonas pálidas debido a la densa fibrosis producida. Los pacientes pueden relatar sensación de quemazón generalizada y limitación de la apertura bucal. La FSO es una enfermedad exclusiva para consumidores de productos a base de Betel, siendo las poblaciones de la India y del sudeste asiático las más afectadas (4, $8,30,31,32$ ).

El Betel Quid consiste en una hoja de Betel enrollada en una mezcla de nuez de Areca, cal hidratada, tabaco y especias. El rol de la nuez de areca en el desarrollo de la FSO es fundamental, múltiples estudios sobre animales comprobaron sus propiedades mutagénicas, genotóxicas y carcinogénicas (32); sin embargo, a pesar de su comprobado efecto negativo, se ha vuelto una parte importante en la cultura de los países que la usan $(30,31,33)$.

\section{Liquen plano}

Se trata de un desorden inflamatorio crónico, el cual, está caracterizado por la presencia de lesiones blancas simétricas y bilaterales similares a líneas o estrías, siendo las zonas más afectadas la lengua, mucosa bu- cal, labios y encías $(34,35,36,37,38,39)$. Si bien la literatura relata una mayor prevalencia en pacientes femeninas de mediana a avanzada edad, en un estudio realizado por Aghbari se halló un mayor número de casos en pacientes masculinos (34). Otra forma clínica descrita por Andreasen en 1964 es el Liquen Plano Erosivo o Eritematoso. Esta variación se manifiesta como áreas rosáceas y eritematosas que pueden tener solución de continuidad, dando lugar a úlceras crónicas, únicas o múltiples. En esta situación, el paciente manifiesta dolor, a veces intenso, y una gran incapacidad funcional $(40,41)$.

El tratamiento recomendado para esta lesión es el uso de corticoides tópicos o sistémicos. Los inhibidores de la calcineurina son también recomendados en caso exista resistencia a los corticoides $(35,37)$. Un seguimiento constante del paciente con liquen plano es primordial para prever la transformación maligna de la lesión (34).

\section{Leucoqueratosis nicotínica}

También conocida como Estomatitis Nicotínica, es un trastorno benigno de la mucosa masticatoria. Se observa clínicamente como pápulas blanquecinas con centros punteados rojizos en la región posterior del paladar duro. Su etiología está directamente relacionada al hábito de fumar y su sintomatología es indolora. Sin embargo, los químicos encontrados en el cigarrillo no son los únicos factores causales de este trastorno. Existe un hábito conocido como fumar de manera invertida en el cual la parte encendida del cigarro se coloca por dentro de la boca, pudiendo alcanzar temperaturas de hasta $120^{\circ} \mathrm{C}$ en el aire intraoral $(42,43)$.

Si bien este trastorno se produce por la exposición crónica al calor y no únicamente por los químicos del cigarro, un consumo y hábito prolongado puede aumentar el riesgo de generar un COCE. Su tratamiento se basa en la suspensión del hábito y controles periódicos $(42,43)$.

\section{DISCUSIÓN}

Si bien cada lesión mencionada en este trabajo cuenta con antecedentes registrados y artículos publicados, algunas de estas aún son cuestionadas por algunos especialistas que consideran que la forma en la que se realizaron los estudios antiguos no fueron adecuados. Es el caso de Müller en el 2017 que puso en cuestión el potencial de malignidad del liquen plano, esto 
debido a una falta en el criterio de diagnóstico que tuvieron estudios pasados sobre esta lesión (38); por ejemplo, en 1999, Van Der Meij alertó que casi dos tercios de los relatos de casos publicados sobre esta lesión tuvieron una documentación inadecuada (44), hecho que pone en duda si es que realmente las lesiones relatadas correspondían a un diagnóstico de Liquen plano y no a otra lesión blanca. No fue hasta el 2003 que la Organización Mundial de la Salud ajustó los criterios de diagnóstico para poder diferenciar al Liquen Plano de otras lesiones liquenoides $(6,34,35$, $38,45)$.

Lo mismo pasa con el caso de la eritroplasia la cual si bien hay artículos como el de Nielsen (17), que confirman su relación al VPH, otros estudios, como lo es el caso de Holmstrup en el año 2017, pone en cuestionamiento a esta hipótesis debido a que la población utilizada por Nielsen no es lo suficientemente grande como para crear una relación (14). Debido a la falta de evidencia sobre el tema, esta asociación es incierta. Motivo por el cual son necesarios más estudios sobre el caso $(1,14,17)$.

Estos tipos de cuestionamientos son importantes para mantener alerta a los investigadores acerca de la metodología de cada artículo utilizado como base teórica y resalta la importancia de todas las partes de un trabajo de investigación el cual no debe centrarse únicamente en el resultado o en las conclusiones.

Este trabajo busca dejar en claro que tipo de responsabilidad y funciones debe asumir un odontólogo general frente a un hallazgo de DOPMs en la consulta cotidiana. Según la Academia Americana de Patología Oral y Maxilofacial, se recomienda que toda anormalidad en los tejidos debería ser sometida adecuadamente a una evaluación y análisis al microscopio (46, 47). Creemos que frente la aparición de una lesión de este tipo, la conducta del odontólogo general se debe limitar a detectar, informar y direccionar al paciente hacia un especialista del área de Patología Oral, capacitado en la prevención, diagnóstico clínico e histopatológico y tratamiento no quirúrgico de los DOPMs, o de un cirujano Buco Maxilofacial en el caso de ser necesaria una intervención quirúrgica de patologías de todo nivel de complejidad en la región de los maxilares. En caso la DOPM avance a una condición maligna, serán los especialistas quienes determinen su derivación a un Oncólogo.

Finalmente, podemos concluir cuatro puntos principales:

Adoptando medidas preventivas, así como también realizar un meticuloso examen clínico sobre tejidos blandos, se puede facilitar la detección temprana de DOPMs.

La biopsia por incisión, seguida por un estudio histopatológico, es considerada un Gold Standard en el diagnóstico de muchos desórdenes y enfermedades de la mucosa oral, incluyendo los DOPMs.

Tratar una lesión o controlar hábitos que pudieran potenciar las condiciones cancerígenas de una DOPM puede evitar en gran mayoría el desarrollo de tumores malignos.

El aventurarse a realizar un tratamiento sin la debida preparación ni experiencia, no solo puede llevar a un fracaso terapéutico en estos casos, sino también a empeorar el pronóstico del paciente.

\section{Correspondencia:}

Marcos Felipe Iparraguirre Nuñovero

Correo electrónico: endoiparraguirre@gmail.com

\section{REFERENCIAS BIBLIOGRÁFICAS}

1. Kalavrezos N, Scully C. Mouth Cancer for Clinicians Part 6 : Potentially Malignant Disorders. Dent Update. 2015;42(9):866-77.

2. Martínez C, Hernández M, Martínez B, Adorno D. Frecuencia de displasia epitelial y carcinoma escamoso en mucosa oral y orofaríngea en Chile, entre los años 1990 y 2009. Rev Med Chil. 2016;144(2):169-74.

3. Niaz K, Maqbool F, Khan F, Bahadar H, Hassan FI, Abdollahi M. Smokeless tobacco (paan and gutkha) consumption, prevalence, and contribution to oral cancer. Epidemiol Health. 2017;39e:1-11.

4. Tommasi AF, Tommasi MH. Lesões e condições Cancerizáveis. In: Diagnóstico em patologia bucal. 4a ed. Rio de Janeiro: Elsevier Ltd; 2015. p. 307-62.

5. Dost F, Lê Cao K, Ford PJ, Ades C, Farah CS. Malignant transformation of oral epithelial dysplasia: a real world evaluation of histopathological grading. Oral Surg Oral Med Oral Pathol Oral Radiol. 2013;117(3):343-52.

6. Majchrzak E, Szybiak B, Wegner A, Pienkowski P, Pazdrowski J, Luczewski L, et al. Oral cavity and oropharyngeal squamous cell carcinoma in young adults: a review of the literature. Radiol Oncol. 2014;48(1):1-10.

7. Carrard VC, Van Der Waal I. A clinical diagnosis of oral leukoplakia ; A guide for dentists. Med Oral Patol Oral Cir Bucal. 2018;23(1):59-64.

8. Neville B, Damm D, Allen C, Chi A. Patologia Epitelial. In: Patologia Oral e Maxilofacial. 4a ed. Rio de Janeiro: Elsevier Ltd; 2016. p. 349-70.

9. Regezi JA, Sciubba JJ, Jordan RCK. Lesões 
Neoplásicas e Pré-Neoplásicas. In: Regezi J, Sciubba J, Jordan R. Patologia Oral Correlações clinicopatológicas. 6a ed. Rio de Janeiro: Elsevier Brasil; 2013. p. 90-110.

10. Warnakulasuriya S, Ariyawardana A. Malignant transformation of oral leukoplakia: a systematic review of observational studies. J Oral Pathol Med. 2016;45(3):155-66.

11. Munde A, Karle R. Proliferative verrucous leukoplakia: An update. J Cancer Res Ther. 2016;12(2):469-73.

12. Villa A, Woo S Bin. Leukoplakia-A Diagnostic and Management Algorithm. J Oral Maxillofac Surg. 2016;75(4):723-34.

13. Yang S, Lee Y, Chang L, Hwang C, Luo C, Chen T. Clinical characteristics of narrow-band imaging of oral erythroplakia and its correlation with pathology. BMC Cancer. 2015;15(1):1-8.

14. Holmstrup P. Oral erythroplakia- What is it? Oral Dis. 2018;24(1-2):138-43.

15. Mello FW, Melo G, Meurer MI, Riet E, Rivero C. Intraoral Potentially Malignant Disorders in a Brazilian Oral Pathology Service : Epidemiological , Clinical, and Histopathological Findings. J Oncol. 2018;2018:1-7.

16. Yang S, Lee Y, Chang L, Hsieh T, Chen T. Outcome of excision of oral erythroplakia. Br J Oral Maxillofac Surg. 2015;53(2):142-7.

17. Nielsen H, Norrild B, Vedtofte P, Praetorius F, Reibel J, Holmstrup P. Human papillomavirus in oral premalignant lesions. Eur J Cancer Oral Oncol. 1996; 32:264-270.

18. Warnakulasuriya S. Clinical features and presentation of oral potentially malignant disorders. Oral Surg Oral Med Oral Pathol Oral Radiol. 2018;125(6):582-90. Doi: 10.1016/j.oooo.2018.03.011

19. Awadallah M, Idle M, Patel K, Kademani D. Management update of potentially premalignant oral epithelial lesions. Oral Surg Oral Med Oral Pathol Oral Radiol. 2018;125(6):628-36. doi: 10.1016/j. oooo.2018.03.010

20. Rhodus NL, Kerr AR, Patel K. Oral cancer: leukoplakia, premalignancy, and squamous cell carcinoma. Dent Clin North Am. 2014;58(2):315-40.

21. Choi SH, Kim KH, Song K. Efficacy of ablative fractional laser-assisted photodynamic therapy for the treatment of actinic cheilitis : 12-month follow-up results of a prospective, randomized, comparative trial. Br J Dermatol. 2015;173(1):184-91.

22. Flórez Á, Batalla A, Torre C De, Batalla A, Torre C De. Management of actinic cheilitis using ingenol mebutate gel : A report of seven cases seven cases. J Dermatolog Treat. 2016;28(2):149-51.

23. Ito $\mathrm{T}$, Natsuga $\mathrm{K}$, Tanimura S, Aoyagi S, Shimizu H. Dermoscopic Features of Plasma Cell Cheilitis and Actinic Cheilitis. Acta Derm Venereol. 2014;94(5):593-4.

24. Chavez YN, Torezan LA, Lourenço S, Neto CF. Evaluation of the efficacy of photodynamic therapy for the treatment of actinic cheilitis. Photodermatol Photoimmunol Photomed. 2016;33(1):14-21.

25. Hernández C, Fuentes $\mathrm{B}$, Cartes-Velazques R. Queilitis actínica: aspectos histológicos, clínicos y epidemiológicos Actinic cheilitis: histological , clinical and. Rev Cubana Estomatol. 2018;53(2):4555.

26. Tzika E, Masouyé I, Mühlstädt M, Laffitte E. Ingenol Mebutate for Recalcitrant Chronic Actinic Cheilitis. Dermatology. 2016;232(s1):1-3.

27. Alawi F. Pigmented Lesions of the Oral Cavity: An Update. Dent Clin NA. 2013;57(4):699-710.

28. Pennacchiotti G, Oviedo C, Ortega-pinto A. Solitary pigmented lesions in oral mucosa in Latin American children : A case series. Pediatr Dermatol. 2018;35(3):374-7.

29. Liang Z, Xu S, Jiang L, Zhao C, Sun X, Qin G. Case Report Scalp junctional nevus with malignant transformation ( melanoma ) metastatic to parotid lymph node region, cervical lymph nodes and the back : a case report and review of literature. Int J Clin Exp Pathol. 2015;8(1):954-9.

30. Chitguppi C, Brar T. Paediatric oral submucous fibrosis- The neglected pre-malignancy of childhood. Int J Pediatr Otorhinolaryngol. 2017;97:55-60.

31. Ekanayaka RP, Tilakaratne WM. Oral submucous fibrosis: Review on mechanisms of malignant transformation. Oral Surg Oral Med Oral Pathol Oral Radiol. 2016;122(2):192-9.

32. Ray JG, Ranganathan K, Chattopadhyay A. Malignant transformation of oral submucous fibrosis: Overview of histopathological aspects. Oral Surg Oral Med Oral Pathol Oral Radiol. 2016;122(2):200-9.

33. Anuradha A, Patil B, Asha VR. Evaluation of efficacy of aloe vera in the treatment of oral submucous fibrosis - a clinical study. J Oral Pathol Med. 2016; 46(1):50-5.

34. Aghbari SMH, Abushouk AI, Attia A, et al. Malignant transformation of oral lichen planus and oral lichenoid lesions : A meta-analysis of 20095 patient data. Oral Oncol. 2017;68:92-102.

35. Alrashdan MS, Cirillo N, Mccullough M. Oral lichen planus : a literature review and update. Arch Dermatol Res. 2016;308(8):539-51.

36. Cascone M, Celentano A, Adamo D, Leuci S, Ruoppo E, Mignogna MD. Oral lichen planus in childhood : a case series. Int J Dermatol. 2017;56(6):641-52.

37. Kurago ZB. Etiology and pathogenesis of oral lichen planus: an overview. Oral Surg Oral Med Oral Pathol Oral Radiol. 2016;122(1):72-80.

38. Müller S. Oral lichenoid lesions : distinguishing 
the benign from the deadly. Mod Pathol. 2017;30(s1):S54-67.

39. Peng Q, Zhang J, Ye X, Zhou G. Tumor-like microenvironment in oral lichen planus: evidence of malignant transformation? Tumor-like microenvironment in oral lichen planus : evidence of malignant. Expert Rev Clin Immunol. 2017;13(6):1-9.

40. Andreasen, J, O. Oral lichen planus: I. A clinical evaluation of 115 cases. Oral Surg Oral Med Oral Pathol. 1968;25(1): 31-42.

41. Blanco A, Otero E, Peñamaría M, Diniz $M$. Diagnóstico del liquen plano oral. Avan en odonto. 2008;24(1): 11-31.

42. Quiñonez L, Ulloa L. Estomatitis nicotínica asociada al hábito de fumar cigarro invertido en paciente ecuatoriana. Acta Odonto Colomb. 2019;9(2): 103-111.

43. Cawson R, Odell W. Fundamentos de medicina y patología oral. 9a edición. Barcelona: Editorial Elsevier; 2018. p. 293.

44. Van Dis M, van der Meij EH, Schepman KP, Smeele
LE, van der Wal JE. A review of the recent literature regarding malignant transformation of oral lichen planus. Oral Surg, Oral Med, Oral Pathol, Oral Radiol Endodontol. 1999;88:307-10.

45. Radwan-Oczko M, Zwyrtek E, Owczarek JE, Szczesniak D. Psychopathological profile and quality of life of patients with oral lichen planus. J Appl Oral Sci. 2018;26:1-9.

46. Melrose R, Handlers P, Kerpel S, Summerlin J, Tomich $\mathrm{J}$. The use of biopsy in dental practice. The position of the American Academy of Oral and Maxillofacial Pathology. Gen dent. 2007;55(5): 457-61.

47. Logan M, Goss A. Biopsy of the oral mucosa and use of histopathology services. Austr dental J. 2010;55, 9-13.

Recibido: 21-04-20

Aceptado: 23-09-20 